\title{
Topical application of aminoglycoside antibiotics enhances host resistance to viral infections in a microbiota-independent manner
}

\author{
Smita Gopinath ${ }^{1,2}$, Myoungjoo V. Kim², Tasfia Rakib², Patrick W. Wong ${ }^{2}$, Michael van \\ Zandt $^{3}$, Natasha A. Barry ${ }^{4}$, Tsuneyasu Kaisho ${ }^{5}$, Andrew L. Goodman ${ }^{1,4}$, and Akiko \\ Iwasaki ${ }^{1,2, *}$ \\ ${ }^{1}$ Howard Hughes Medical Institute (New Haven, CT, (USA)) \\ ${ }^{2}$ Department of Immunobiology, Yale University, (New Haven, CT, (USA)) \\ ${ }^{3}$ New England Discovery Partners (Branford, CT, (USA)) \\ ${ }^{4}$ Department of Microbial Pathogenesis and Microbial Sciences Institute, Yale University (New \\ Haven, CT, (USA)) \\ ${ }^{5}$ Department of Immunology, Institute of Advanced Medicine, Wakayama Medical University, \\ Kimiidera 811-1 (Wakayama 641-8509 (Japan))
}

\section{Abstract}

\begin{abstract}
Antibiotics are widely used to treat infections in humans. However, the impact of antibiotic use on host cells is understudied. Here we identify an antiviral effect of commonly used aminoglycoside antibiotics. We show that topical mucosal application of aminoglycosides prophylactically increased host resistance to a broad range of viral infections including herpes simplex viruses, influenza A virus and Zika virus. Aminoglycoside treatment also reduced viral replication in primary human cells. This antiviral activity was independent of the microbiota as aminoglycoside treatment protected germ-free mice. Microarray analysis uncovered a marked upregulation of transcripts for interferon-stimulated genes (ISGs) following aminoglycoside application. ISG induction was mediated by TLR3, and required TIR-domain-containing adapter-inducing interferon- $\beta$ (TRIF), signaling adaptor, and interferon regulatory factors 3 (IRF3) and IRF7, transcription factors that promote ISG expression. XCR1+ dendritic cells, which uniquely express TLR3, were recruited to the vaginal mucosa upon aminoglycoside treatment and were required for
\end{abstract}

\footnotetext{
Users may view, print, copy, and download text and data-mine the content in such documents, for the purposes of academic research, subject always to the full Conditions of use: http://www.nature.com/authors/editorial_policies/license.html\#terms

*To whom correspondence should be addressed: Akiko Iwasaki, Ph.D., Department of Immunobiology, Yale University School of Medicine, 300 Cedar Street, New Haven, CT 06520, Phone: (203) 785-2919, FAX: (203) 785-4972, akiko.iwasaki@ yale.edu. Correspondence and requests for materials should be addressed to Akiko Iwasaki

Contributions:

SG and AI planned the project, designed the experiments, interpreted the data and wrote the paper. SG, MK, TR, PW designed and carried out the experiments. NB and AG provided reagents and help with germ-free experiments. TK, MZ and AG provided reagents and feedback.

Competing Interests

The authors have no competing interest to report.
} 
ISG induction. These results highlight an unexpected ability of aminoglycoside antibiotics to confer broad antiviral resistance in vivo.

\section{Keywords}

aminoglycosides; pattern recognition receptors; antiviral; interferon; herpes simplex virus; influenza virus; mucosal immunity

\section{Main}

Antibiotics comprise a large and complex group of compounds typically secreted by bacteria targeting other prokaryotes. Antibiotics have revolutionized medicine since their discovery. However, overuse and misuse of antibiotics have led to the emergence of resistant bacteria in humans and in livestock. Current medicine faces a dire threat of infections caused by antibiotic resistant bacteria. In addition to its microbicidal activities, antibiotics can also directly affect mammalian cells in a variety of ways. Antibiotics have been reported to directly inhibit eukaryotic translation ${ }^{1}$, inhibit mitochondrial function ${ }^{2,3}$ and induce changes in mammalian metabolic pathways ${ }^{4}$. There is a critical need to better understand the host effects of commonly used antibiotics.

Here, we found that topical delivery of aminoglycoside antibiotics induced significant alteration of host gene expression, increasing expression of antiviral interferon-stimulated genes (ISGs). Aminoglycoside-mediated ISG induction resulted in significant protection against RNA and DNA viruses and was independent of the host microbiota. ISG expression was dependent on TLR3 and downstream signaling pathway, and robust recruitment of TLR3-expressing XCR1+ dendritic cells to the vaginal mucosa. Our results reveal an unexpected induction of antiviral state by commonly used aminoglycoside antibiotics.

To investigate the effect of local, vaginal, antibiotic treatment on genital herpes virus infection, we treated mice daily with an antibiotic cocktail (composed of $0.5 \mathrm{mg}$ each of ampicillin, neomycin and vancomycin and $0.012 \mathrm{mg}$ metronidazole) administered intravaginally. All mice were first injected subcutaneously with Depo-provera to synchronize estrus cycles and increase HSV-2 infectivity ${ }^{5}$. After a week of daily antibiotic treatment, mice were infected intravaginally with HSV-2. After genital infection, HSV-2 undergoes multiple rounds of local replication in the vaginal mucosa which can be quantified by plaque assays for infectious virus in the vaginal wash ${ }^{6,7}$. In mice, by four days post infection, the virus travels to the dorsal root ganglion where it can spread to fresh epithelial sites resulting in disease symptoms including hair loss and hind limb paralysis which can be quantified using a clinical scoring system ${ }^{8}$. Neural spread of HSV-2 is required for disease as herpes viruses unable to replicate in neurons are unable to cause neurological symptoms and morbidity ${ }^{9}$. Antibiotic-treated mice had significantly decreased vaginal viral titers and displayed fewer clinical symptoms of genital herpes infection indicating suppression of both early and late stages of HSV-2 infection (Fig. 1a,b). Vaginal viral titers of antibiotic pretreated mice were only suppressed early in infection (days 1-3), eventually reaching levels similar to control mice (Supplementary Fig. 1a). Reduced viral titers were also observed in the dorsal root ganglion upon antibiotic treatment (Supplementary Fig. 1c). 
Importantly, this transient suppression was sufficient to significantly protect mice against morbidity as antibiotic pre-treated mice displayed little to no disease pathology (Fig. 1a).

To determine which of the antibiotics in the cocktail are required for protection against HSV infection, we treated mice singly with each antibiotic. We excluded metronidazole as it was present in very low quantities in the cocktail. Of the four antibiotics tested, neomycin alone recapitulated the host protection observed with the full antibiotic cocktail with disease scores (Fig. 1c) and vaginal viral titers (Fig. 1d, Supplementary Fig. 1b) significantly lower than both PBS controls and the other single antibiotic-treated mice (Fig. 1c). Thus, neomycin is the antiviral ingredient in the antibiotic cocktail.

Antibiotic-mediated effects on host immunity are often attributed to reduction or dysbiosis of relevant commensal bacterial communities. To determine if neomycin-mediated antiviral effect requires the host microbiota, we treated germ-free mice intravaginally with neomycin. Notably, neomycin treatment of germ-free hosts also resulted in significant protection against genital HSV-2 infection. Neomycin-treated germ-free mice displayed no viral disease pathology (Fig. 1e) and had no detectable replicating virus in the vaginal mucosa (Fig. 1f) indicating that neomycin-mediated antiviral effect is independent of live or dead vaginal commensals, or microbiota in general. To determine if neomycin treatment had a therapeutic effect, HSV-2 infected mice were treated with neomycin at 4, 24,48 and 72 hours post infection. Post exposure neomycin treatment resulted in lower viral titers (Fig. 1h, Supplementary Fig. 1d) and reduced disease scores (Fig. 1g). Post exposure neomycin starting at 24 hours post infection resulted in variable protection (Supplementary Fig. 1e,f). These data indicated that vaginal application of neomycin before or shortly after exposure reduces HSV-2 infection and disease, independent of commensal bacteria.

To elucidate the mechanisms underlying neomycin-mediated host resistance against HSV-2, we analyzed vaginal gene transcription prior to infection. We identified over a hundred significantly upregulated genes (Fig. 2a; Supplementary Table 1). No genes were significantly downregulated in expression. Using Ingenuity pathway analysis, we found that genes in the type I interferon (IFN) pathway were heavily enriched (Fig. 2b), with over 30\% of the upregulated genes falling within this pathway. We independently confirmed the upregulation of a subset of IFN-stimulated-genes (ISGs) using RT-qPCR (Fig. 2c). ISG expression was also increased in germ-free mice upon neomycin treatment (Supplementary Fig. 2). Neomycin-induced ISG expression was rapid, as a single treatment was sufficient to significantly increase ISG expression $2-5$ fold though not to the levels observed after a week's treatment (8-10 fold) (Supplementary Fig. 3a). Neomycin-mediated ISG induction was also dose-dependent as increased amounts of neomycin correspondingly increased ISG expression (Supplementary Fig. 3a). Significant ISG induction was maintained up to 3 days after neomycin treatment and was only partially lost by 1 week, with 5 of 8 genes still significantly upregulated (Supplementary Fig. 3c). Finally, neomycin-mediated ISG induction was restricted to the site of application, as we observed no upregulation of ISG expression in the lungs of neomycin-treated mice (Supplementary Fig. 3d).

Neomycin is a member of a large and diverse group of aminoglycosides, many of which are commonly used to treat bacterial infection. To determine if ISG induction was common 
across the aminoglycoside family, we treated mice with a panel of structurally diverse aminoglycosides for 1-2 days. Five out of the seven aminoglycosides we tested increased ISG expression to varying levels of significance upon application to the vaginal mucosa (Fig. 2d). The two non-inducers were streptomycin, which contains a streptamine core distinct from the other 2-deoxystreptamine-containing aminoglycosides, and amikacin, a kanamycin homolog that contains an addition L-haba side chain ${ }^{10}$. Thus, the majority of the aminoglycosides tested induce ISG expression upon application to the vaginal mucosa. Since aminoglycosides mediate their antibiotic activity by binding ribosomal RNA, we tested if this ISG-induction was observed in other ribosome-targeting antibiotics ${ }^{11}$. Mice were treated intravaginally with tetracycline or chloramphenicol, compounds that inhibit bacterial protein synthesis by binding the 30s and 50s ribosome subunit respectively 11 (Supplementary Fig. 4a,b). Neither compound induced high levels of ISGs, suggesting that ISG induction is not a common property of antibiotics that target the ribosome. Previous studies have reported increased mitochondrial dysfunction and a loss of mitochondria in mammalian cells ${ }^{2,12}$ upon treatment with multiple classes of antibiotics. We observed no reduction in total mitochondrial DNA in neomycin-treated vaginal tissues (Supplementary Fig. 4c).

To determine the link between ISG expression and antiviral protection, we compared the ability of ISG-inducing and ISG non-inducing aminoglycosides to protect mice against HSV-2 infection. We selected two ISG-inducing aminoglycosides (kasugamycin, sisomicin) as well as the two ISG non-inducing aminoglycosides (amikacin, streptomycin). Mice pretreated with ISG non-inducers were not protected against HSV-2 infection (Fig. 2e,f). Kasugamycin and sisomicin treatment however, resulted in significant reduction in vaginal viral titers and disease scores, displaying equivalent levels of antiviral protection to neomycin (Fig. 2e,f). These data indicated that ISG induction by aminoglycosides corresponds to their ability to protect mice against viral challenge.

Next, we examined if aminoglycoside induction of antiviral immunity was specific to the vaginal tract or if similar protection could be observed in other mucosal surfaces such as the respiratory tract. A single intranasal dose of neomycin sufficed to significantly upregulate ISG expression in the lung (Fig. 3a). To determine if this ISG induction was functional, we used an Mx1 congenic mouse model of influenza infection ${ }^{13}$. Many inbred mouse strains, including C57BL/6, lack Mx1 (Ref. ${ }^{14}$ ). $M x 1$ encodes myxovirus resistance protein 1, a dynamin-like GTPase that blocks primary transcription of influenza by binding to viral nucleoproteins ${ }^{15}$. Mx1 is also an ISG and is induced by neomycin treatment (Fig. 3a). In mice lacking Mx1, innate resistance to influenza is abrogated, and they rely on adaptive immunity. We previously showed that oral neomycin treatment renders Mx1 deficient mice susceptible to influenza disease because it depletes gut commensal bacteria that normally activate dendritic cells that prime CD8 T cells ${ }^{16}$. In contrast, the use of Mx1 congenic mice allows us to study the ability of intranasal neomycin to elicit innate antiviral resistance through induction of ISGs including $M x 1$. We infected neomycin-pretreated Mx1 congenic mice with a highly virulent influenza A virus A/PR8 (hvPR8), which was selected for its ability to replicate rapidly even in the presence of Mx1 (Ref. ${ }^{17}$ ). A single dose of neomycin was sufficient to significantly increase survival following challenge with an otherwise lethal dose of hvPR8, with $40 \%$ of mice protected (Fig. 3b). Intranasal treatment with the ISG non- 
inducer streptomycin, failed to induce ISG expression in the lung (Fig. 3a) and Mx1 congenic mice pretreated with streptomycin were not protected against influenza infection (Fig. 3b).

We also tested the ability of aminoglycosides to protect against another RNA virus infection - Zika virus (ZIKV). ZIKV replicates in the vagina of wild-type mice and is controlled by IRF3 and IRF7-dependent type I IFN secretion and type I IFN receptor (IFNAR) signaling 18. Given the ability of aminoglycosides to induce ISGs, we examined if aminoglycosidetreated hosts are protected against ZIKV infection. We found that kasugamycin and neomycin treatment resulted in significantly lower levels of ZIKV RNA in the vaginal mucosa 1-2 days post infection (Fig. 3c). While vaginal ZIKV titers in neomycin-treated mice reached those in PBS-treated controls by day 3 post infection, ZIKV replication in kasugamycin-treated mice remained significantly lower in a large proportion of mice in this group (Fig. 3c).

To extend our findings to human cells, we treated primary peripheral blood monocytes obtained from healthy donors with kasugamycin. Kasugamycin treatment significantly increased ISG expression in human monocytes similar to Poly I:C treatment (Fig. 3d). Six hours of kasugamycin treatment was sufficient to induce robust ISG expression and significantly reduce replication of influenza A virus in monocytes (Fig. 3e). Collectively, our results demonstrate that aminoglycosides provide antiviral protection against a diverse set of DNA and RNA viruses: HSV-2, influenza A virus and ZIKV.

Since neomycin treatment resulted in increased expression of genes in nucleic acid sensing pathways, we asked if cytosolic or endosomal nucleic acid sensors were involved. To test the requirement of cytosolic nucleic acid sensors, mice lacking the RIG-I like receptor signaling adaptor Mavs, or the cytosolic DNA sensor cGAS (Mb21d1), and its downstream adapter STING (Tmem173) were treated with neomycin or PBS and subsequent vaginal gene expression was examined. While neomycin induction of ISG expression was intact in cGAS -/- or $\mathrm{STING}^{-/-}$mice, neomycin-treated $\mathrm{MAVS}^{-/-}$hosts showed relatively lower levels of ISG induction (Supplementary Fig. 5a,d). Infection of neomycin-treated $\mathrm{MAVS}^{-/}$hosts resulted in reduced protection (Supplementary Fig. 5b,c). By contrast, neomycin-treatment of STING ${ }^{-/}$mice resulted in robust antiviral protection with significantly suppressed viral replication and no disease pathology (Supplementary Fig. 5e,f). These results indicate that the cytosolic DNA sensor signaling pathway is dispensable for ISG induction by neomycin, while RNA sensor signaling pathways play a partial role in aminoglycoside-mediated ISG induction.

We next investigated the role of endosomal RNA sensors. TLR7 signaling was dispensable for aminoglycoside-mediated antiviral protection (Supplementary Fig. 6a,b). However, we found that treatment of $T I r 3^{-1-}$ mice with neomycin resulted in no ISG induction (Fig. 4a) and was accompanied by significant loss of protection against HSV-2 infection (Fig. 4b,c). TLR3 signals via the adaptor protein TRIF ${ }^{19}$. To confirm the activation of TLR3 signaling pathway in aminoglycoside-mediated antiviral protection, we treated 
Trif $^{/-}$mice with neomycin. ISG induction was diminished in Trif $^{/-}$mice as compared to neomycin-treated WT mice (Fig. 4d). Similar to TIr $3^{-/-}$hosts, we observed a lack of protection against genital HSV-2 infection. Neomycin-treated Trif $^{-/-}$mice had equivalent vaginal viral titers and similar disease scores as PBS controls (Fig. 4e,f). Finally, we investigated the role of transcription factors downstream of TLR3 and TRIF signaling. ISGs downstream of cytosolic and endosomal nucleic acid sensors require IRF3 and IRF7 for induction ${ }^{20}$ so we investigated neomycin-mediated ISG expression in Irf ${ }^{-1-} \mathrm{IrfT}^{-1-}$ mice. Similar to Trif ${ }^{-1-}$ and $T I r 3^{-1-}$ mice, no upregulation of ISGs was observed in neomycintreated $I r 3^{-1-} I r f 7^{-1-}$ mice (Fig. $4 \mathrm{~g}$ ) and this lack of ISG induction correlated with the lack of protection against HSV-2 infection (Fig. 4h,i). Induction of ISGs via IRF3 and IRF7 often requires signaling through the type I IFN receptor (IFNAR) ${ }^{21}$ but IFN-independent ISG induction has also been reported ${ }^{22}$. Neomycin-mediated ISG induction was intact in Ifnar $1^{-1-}$ mice suggesting that IFNAR signaling is dispensable (Supplementary Fig. 7a). However, since basal ISG expression was much lower in Ifnar ${ }^{{ }^{-1}}$ mice, neomycin treatment only increased gene expression to those of untreated WT mice and thus neomycin treatment was not accompanied by significant antiviral protection (Supplementary Fig. 7b,c). Collectively, our results suggest that neomycin induces ISG expression via activation of TLR3-TRIF-IRF3/7 signaling pathway to confer protection against HSV-2.

We next examined the cell types responsible for ISG induction and antiviral protection in the vaginal mucosa upon neomycin treatment. The vaginal mucosa is composed of stratified squamous epithelial cells, small numbers of resident leukocytes and circulating leukocytes. We investigated whether ISG induction by neomycin requires tissue resident cells or circulating leukocytes. To determine if circulating leukocytes were responsible for increased ISG expression, we blocked cellular recruitment by treating mice intravaginally with pertussis toxin. Pertussis toxin (PTX) blocks Gi-protein coupled receptor signaling, thereby preventing most chemokine-mediated cellular recruitment to the vaginal mucosa when applied intravaginally ${ }^{23}$. Treatment with PTX ablated the protective effect of neomycin as mice treated with both pertussis toxin and neomycin prior to infection had significantly reduced vaginal ISG expression compared to PTX-untreated mice (Fig. 5a). This absence of ISG expression resulted in significantly higher levels of vaginal viral titers and an increase in disease pathology upon HSV-2 infection (Fig. 5b,c). These results suggested that cellular recruitment to the vagina is a prerequisite for enhanced ISG induction and protection against HSV-2 conferred by topical neomycin treatment.

To determine the specific cell types recruited to the vaginal mucosa by neomycin, we compared the cellular composition of the vaginal mucosa in mice treated with neomycin and those treated with neomycin and PTX. Monocytes (CD11b+), monocyte-derived DCs $(\mathrm{CD} 11 \mathrm{~b}+\mathrm{CD} 11 \mathrm{c}+)$ and classical DCs (CD11c+), were significantly increased upon neomycin treatment (Fig. 5d,e). However, PTX treatment of neomycin-inoculated mice resulted in significant blockade of $\mathrm{CD} 11 \mathrm{c}+$ and $\mathrm{CD} 11 \mathrm{~b}+\mathrm{CD} 11 \mathrm{c}+$ cells (Fig. 5e), suggesting that one or both of these cell types might be responsible for the neomycin-mediated ISG induction.

To test this hypothesis, we isolated CD11c+ cells from the vaginal tissue of neomycintreated and control mice and found significant increases in transcripts of ISGs 
(Supplementary Fig. 8). Likewise, these recruited CD11c+ cells were also found to express IFN $\beta$ unlike plasmacytoid DCs (Supplementary Fig. 9a-c). To determine whether DCs are required for the increase in ISG expression following neomycin application, we treated CD11c-DTR mice with diphtheria toxin to deplete DCs and then administered neomycin intravaginally. In the absence of DCs, neomycin treatment failed to increase vaginal ISG expression (Fig. 5f). Collectively, our results suggest that recruited DCs are necessary for aminoglycoside-mediated ISG expression.

Since both monocyte-derived and classical DCs are recruited to the vaginal mucosa, we wondered if TLR3 expression could be used to identify the specific DC subset involved. It is well known that TLR3 is expressed selectively by the cDC1 and not cDC2 subsets 24,25 . Examining TLR3 expression across all DC subsets using the RNA sequencing datasets deposited in the Immunological Genome project ${ }^{26}$ confirmed a single cDC subset with high levels of TLR3 expression - CD8a+ DCs (cDC1) from the thymus and spleen (Supplementary Fig. 10). In non-lymphoid tissue, these cells are characterized as CD103+ DCs and both subsets express high levels of TLR3 (Ref. ${ }^{27}$ ). Recent studies have identified $\mathrm{XCR} 1$ as a defining cell surface marker for $\mathrm{CDC} 1$ subset $^{28}$. Thus, we measured the recruitment of XCR+CD103+ DCs in the vaginal mucosa. Neomycin treatment resulted in significant recruitment of CD103+ XCR1+ DCs, which was blocked upon co-treatment with pertussis toxin (Fig. 6a,b). To determine if this was the DC subtype responsible for ISG induction by aminoglycosides, we depleted mice of XCR $1+$ DCs ${ }^{29}$ before intravaginal aminoglycoside treatment. Mice lacking XCR1+ DCs showed no induction of ISG expression upon aminoglycoside treatment (Fig. 6c). These data suggested that mucosal application of aminoglycosides induce ISGs in TLR3 and XCR+ DC-dependent manner to confer antiviral protection.

Systemic administration of aminoglycosides has known toxic side-effects including ototoxicity and nephrotoxicity ${ }^{30}$. Aminoglycoside compounds can accumulate in the sensory hair cells of the inner ear causing caspase-mediated cell death which results in irreversible hearing loss ${ }^{31-33}$. While nephrotoxicity can be reversible, significant necrosis can occur resulting in kidney dysfunction ${ }^{34,35}$. However, we observed no toxicity in mice treated with intravaginal aminoglycosides. To determine if intravaginal aminoglycoside treatment resulted in mucosal toxicity, we conducted blinded histological analysis of aminoglycoside-treated and control vaginal tissues which found no histopathological differences between two groups (Supplementary Fig. 11a). Likewise, ex-vivo treatment of splenocytes with aminoglycosides induced robust ISG expression with little accompanying toxicity (Supplementary Fig. 11b-d). Similar to our in-vivo results, depletion of DCs resulted in significant loss of ISG induction (Supplementary Fig. 11e).

Aminoglycosides act by binding bacterial ribosomal RNA, but they also bind mitochondrial and mammalian ribosomal RNA ${ }^{36-38}$. Our data show that aminoglycoside induction of ISG requires TLR3, which is a sensor of dsRNA. We hypothesized that aminoglycosides may induce ISG expression by rendering host RNA more 'visible' to TLR3 in neighboring DCs. To test this, we first treated splenocytes with kasugamycin and washed the cells multiple times to remove extracellular aminoglycosides. Next, we incubated these kasugamycintreated cells with splenic DCs that include XCR1+ DCs from WT and TLR3 knockout mice 
and measured DC-specific ISG expression. Incubation with kasugamycin-treated splenocytes was sufficient to increase ISG expression in WT but not $T I r 3^{-1-}$ DCs (Supplementary Fig. 12).

These data suggest that aminoglycosides may bind to host RNA and render it more potent for TLR3 activation. To test this, we treated splenocytes with a combination of aminoglycosides and dsRNA Poly I:C. We found that 1:1000 ratio of Poly I:C to kasugamycin significantly induced ISG expression at greater levels than either compound alone (Supplementary Fig. 13). This enhancement was dependent on both TLR3 and TRIF signaling (Supplementary Fig. 13). Collectively, these data indicate that at high molar ratio, kasugamicin synergizes with dsRNA to stimulate TLR3.

Our data show an unexpected antiviral effect of aminoglycosides in mucosal tissues. Vaginal application of aminoglycosides conferred resistance against both DNA and RNA viruses. Surprisingly, aminoglycoside-mediated antiviral protection occurred in germ free mice, indicating a microbiome-independent mechanism of resistance. Mucosal aminoglycoside treatment recruited XCR1+ dendritic cells, and induced ISGs expression via TLR3, TRIF and transcription factors IRF3 and IRF7. Finally, this antiviral protection could be extended to both the nasal mucosa and primary human monocytes.

Other antibiotic compounds have been previously reported to inhibit viral replication. Several screens of bioactive compounds for antiviral activity have identified antibiotic compounds including azithromycin and nanchangmycin 39,40 . Azithromycin, a macrolide antibiotic, potently inhibits Zika virus replication in cell culture via an as yet unknown mechanism ${ }^{40}$. Pretreatment of cells with nanchangmycin blocks entry of many flaviviruses including dengue virus and chikungunya virus by inhibiting clathrin-mediated endocytosis 39. These studies collectively highlight the unexpected antiviral functions of antibiotics, albeit through distinct downstream pathways. Our study identifies a class of antibiotics, aminoglycosides, which mediate their antiviral activity by increasing host expression of a broad range of ISGs, potentially reducing the opportunity for viruses to develop resistance. Of note, a previous study demonstrated that macrolide antibiotics induced type I and III IFNs in epithelial cells ${ }^{41}$. Another study showed the ability of anthracyclines (chemotherapy agents that are natural products of Streptomyces bacteria) to also induce ISG expression in cancer cells in a TLR3-dependent manner ${ }^{42}$. Thus, bacterial products with nucleic acid binding capacity may have the ability to trigger TLR3 and possibly other PRRs. Since aminoglycoside-mediated antiviral activity was suppressed at higher levels in Trif ${ }^{\prime-}$ hosts as compared to $T I r 3^{-/}$hosts, it is possible that an alternate sensor upstream of TRIF, such as TLR4, may also play a role.

How do aminoglycosides induce ISGs? Our results indicate that aminoglycoside treatment in the vaginal mucosa increases expression of chemokines and is accompanied by recruitment of monocyte-derived DCs and cDCs. Blocking this recruitment through PTX treatment, or depletion of DCs, aborted the ISG response. With TLR3 as the key sensor required for ISGs and as only cDC1 expresses TLR3 (Ref. ${ }^{27}$ ), we further determined that $\mathrm{XCR} 1+\mathrm{cDC} 1$ were required for ISG induction in the vaginal mucosa. 
Aminoglycosides could be taken up by these DCs via endocytosis ${ }^{43}$ or through TRPV family of ion channels ${ }^{44}$ which are also expressed on DCs ${ }^{45}$. Aminoglycosides may also be taken up by the vaginal epithelial cells via the megalin receptor which has been shown to bind these compounds 46,47 . Aminoglycosides are known for inducing cytotoxicity in specific cell types such as the hair cells of the inner ear and kidney epithelial cells $30,31,34$ but their effect on immune cells has not been well characterized. We observed no inflammation or cytotoxicity in aminoglycoside-treated vaginal tissues (Supplementary Fig. 11a). Of note, incubation of DCs with kasugamycin-treated splenocytes was sufficient to induce ISG expression albeit at lower levels than direct aminoglycoside treatment (Supplementary Fig. 12). As XCR1+DCs are known for their cross-presentation of antigens associated with dead or dying cells ${ }^{48,49}$, it is conceivable that in the vaginal mucosa, phagocytosis of aminoglycoside-containing epithelial cells results in TLR3 activation due to the accumulation of aminoglycoside-bound RNA in the endosome.

The specific RNA bound by the aminoglycoside that triggers TLR3 remains unknown. The interaction of aminoglycosides with mitochondrial ribosomal RNA, which is closely related to bacterial ribosomal RNA, is well characterized and aminoglycosides have also been found to bind mitochondrial ribosomal RNA at multiple sites ${ }^{36,50}$. It is possible that mitochondrial rRNA-bound aminoglycosides might activate TLR3 in XCR1+ DCs in the endosome. Our results show that aminoglycoside and Poly I:C can synergize to induce increased ISG expression (Supplementary Fig. 13). Future studies are needed to identify the manner in which aminoglycoside-bound RNA stimulate TLR3.

Our results demonstrate a surprising and broad antiviral effect of the aminoglycoside family of antibiotics, when applied to mucosal surfaces. However, we do not advocate for use of these compounds as antivirals, as aminoglycoside application is expected to cause local dysbiosis of commensal bacterial community. Understanding the precise mechanism by which aminoglycosides induce TLR3 stimulation will be useful for the future design of novel broad-acting antivirals with no antimicrobicidal activity.

\section{Materials and methods}

\section{Mice}

C57BL/6 (B6; Charles River Laboratories), B6(Cg)-Ifnar1tm1.2Ees/J (Ifnar 1-/-), B6.FVB-

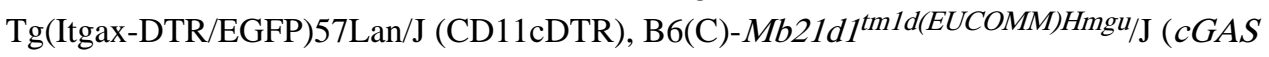
$\left.{ }^{-/-}\right)$, C57BL/6J-Ticam $1^{\text {Lps2 } / \mathrm{J}}\left(\right.$ Trif $\left.^{\prime-}\right)$ mice, C57BL/6J-Tmem1738t/J $\left(\mathrm{STING}^{-/-}\right)$and

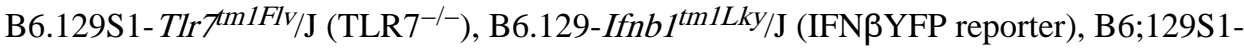
$\operatorname{Tlr}^{\text {tm } 1 F l_{V} / \mathrm{J}}\left(\mathrm{TLR}^{-/-}\right.$) and B6129SF2/J controls were purchased from Jackson Laboratory unless otherwise specified and subsequently bred and housed at Yale University. Irf3 ${ }^{-1-}$ Irf $7^{-1-}$ (Ref. ${ }^{20}$ ) (generous gift by Dr. T. Taniguchi, the University of Tokyo), $\mathrm{MavS}^{-/-}$(Ref. ${ }^{51}$ ) (generous gift by Dr. Z. Chen, UTSW), B6.Cg-Xcr1 ${ }^{\text {tm2(HBEGF/Venus)Ksho (XCR1-DTR) }}$ $\left(\right.$ Ref ${ }^{29}$ ) are previously described. C57BL/6 mice carrying functional Mx1 alleles have were a generous gift by Dr. P. Staeheli ${ }^{52}$ (University Medical Center Freiburg). Mice were maintained in our facility until the ages described. Germ-free Swiss Webster mice were maintained in flexible plastic gnotobiotic isolators with a $12 \mathrm{hr}$ light/dark cycle and provided a standard, autoclaved mouse chow (5K67 LabDiet, Purina) ad libitum and autoclaved water 
for the duration of the experiment. Germ-free status was monitored by culture-based (aerobic and anaerobic culturing) and culture-independent methods (16S-targeted PCR). All procedures used in this study complied with federal and institutional policies of the Yale Animal Care and Use committee.

\section{Viruses}

Wild-type HSV-2 (strain 186syn+) was a kind gift from Dr. David Knipe (Harvard Medical School, Boston, MA). HSV strain was propagated in Vero cells (ATCC CCL-81) as previously described ${ }^{53}$. Highly virulent variant of A/PR8 (Ref. ${ }^{17}$ ) was a generous gift from Dr. P. Staeheli (University Medical Center Freiburg). Influenza virus strain A/PR/8/34 (H1N1) was propagated as previously described ${ }^{16}$. ZIKV Cambodian FSS13025 strain was obtained from the World Reference Center for Emerging Viruses and Arboviruses at University of Texas Medical Branch, Galveston. ZIKV stocks were propagated in C6/36 mosquito cells (Aedes albopictus) (ATCC CRL-1660) as previously described ${ }^{18}$. Cell lines obtained from ATCC were not further authenticated and all cell lines were routinely tested for mycoplasma contamination.

\section{Mouse infections and antibiotic treatment}

C57BL/6 mice between six and seventeen weeks of age were subcutaneously injected with 2 milligrams of Depo-Provera in the neck scruff. Five days after Depo treatment, the mice were vaginally swabbed with a calcium alginate swab (Puritan, Maine) to remove vaginal mucus. The swab was wetted in sterile PBS and blotted on sterile paper to get rid of excess liquid before being used. Ten to 15 microliters of antibiotic or PBS was delivered into the vaginal cavity using a pipette tip. After 2-6 days of daily antibiotic treatment, mice were infected intravaginally with $2 \times 10^{3}$ to $5 \times 10^{3}$ PFU HSV-2, $10^{6}$ PFU HSV-1 or $2.5 \times 10^{5}$ PFU ZIKV. Infections were carried out 24 hours after the last antibiotic treatment. At the time of infection, mice were weighed and subsequently examined at the same time each day to minimize fluctuations in weight due to circadian rhythms. Vaginal viral titers were collected by swabbing mice and flushing the vaginal cavity with 50 microliters of PBS. Mice were monitored daily for signs of inflammatory pathology scored as follows: (0) no inflammation, (1) genital inflammation, (2) genital lesions and hair loss, (3) hunched posture and ruffled fur, (4) hind-limb paralysis and (5) premoribund. Mice were euthanized before reaching a moribund state. Intranasal influenza infections were conducted as described previously ${ }^{16}$. Briefly, mice were anesthetized using a mixture of ketamine and xylazine injected i.p. and infected with $500 \mathrm{PFU}$ highly virulent A/PR8 influenza strain. All mouse experiments were carried out with a minimum of 3-4 mice per treatment group. With the exception of the germ-free mouse infections and experiments with XCR $1^{\mathrm{DTR}}$ mice, all other experiments were independently repeated at least once. These experiments were only conducted once with a total $n=5$. All mouse experiments were conducted on female mice with the exception of intranasal aminoglycoside treatment and influenza infection experiments, which were conducted with both male and female mice. Mice in each experiment were age-matched within a week's time frame and cage-mates were randomly distributed into different treatment groups to control for cage-effects. All mouse experiments were conducted with a minimum of 4-5 mice for infection experiments and a minimum of 3 
mice for gene expression experiments. All animal procedures were performed in compliance with Yale Institutional Animal Care and Use Committee protocols.

\section{Antibiotic treatment}

The antibiotic cocktail consisted of $0.5 \mathrm{mgs}$ Ampicillin, Vancomycin and Neomycin and 0.01 milligram metronidazole in a $15 \mu \mathrm{l}$ volume. For subsequent experiments using neomycin alone, mice were treated with 1 milligram in a volume of $10 \mu$. For intranasal treatment, mice were anesthetized by injecting a mixture of ketamine and xylazine intraperitoneally and $20 \mu \mathrm{L}$ of antibiotic administered dropwise into the nasal cavity using a pipet tip. All antibiotics were obtained from Sigma-Aldrich (Darmstadt, Germany).

\section{Microarray analysis}

Mice were treated daily with neomycin (1mg/day) or PBS for six days and then sacrificed and vaginal tissue harvested. RNA was extracted from the vaginal tissues using an RNeasy extraction kit (Qiagen,CA) and hybridized onto MouseWG-6 v2 Expression arrays (Illumina,CA) at the Yale Center for Genome Analysis. Microarray data was visualized using the ggplot2 (v. 2.2.1) package in $\mathrm{R}$ (v.0.98).

\section{Gene expression analysis}

The following primers were used for mouse ISG expression Hprt (FP: GTTGGATACAGGCCAGACTTTGTTG, RP: GAGGGTAGGCTGGCCTATTGGCT); Zbp1 (FP: TTGCCAATTCAAACGCCATC, RP: CACTTGTTGGAGCAAGGACT); Cxcl10(FP: AGAATGAGGGCCATAGGGAA, RP: CGTGGCAATGATCTCAACAC); Usp18 (FP: CGTGCTTGAGAGGGTCATTTG, RP: GGTCCGGAGTCCACAACTTC); Irf7(FP: TGTAGACGGAGCAATGGCTGAAGT, RP: ATCCCTACGACCGAAATGCTTCCA); Oas12 (FP: GGGAGGTCGTCATCAGCTTC, RP: CCCTTTTGCCCTCTCTGTGG); Oas1a (FP: ATTACCTCCTTCCCGACACC, RP: CAAACTCCACCTCCTGATGC); Rsad2 (Viperin) (FP: AACAGGCTGGTTTGGAGAAG RP: TGCCATTGCTCACTATGCTC); Mx 1 (FP: CCAACTGGAATCCTCCTGGAA, RP: GCCGCACCTTCTCCTCATAG). The following primers were used for human ISG expression: HPRT(FP: TGGTCAGGCAGTATAATCCAAAG, RP: TTTCAAATCCAACAAAGTCTGGC); OAS1 (FP: CTGAGAAGGCAGCTCACGAA, RP: TGTGCTGGGTCAGCAGAATC); OASL (FP: AAAAGAGAGGCCCATCATCCT, RP: CCTCTGCTCCACTGTCAAGT); $C X C L 9$ (FP: AGTGCAAGGAACCCCAGTAG RP: AGGGCTTGGGGCAAATTGTT); CXCL10 (FP:CCACGTGTTGAGATCATTGCT RP: TGCATCGATTTTGCTCCCCT); USP18 (FP:GGCTCCTGAGGCAAATCTGT RP: CAACCAGGCCATGAGGGTAG ); $M X 1$ (FP: AGAGAAGGTGAGAAGCTGATCC; RP: TTCTTCCAGCTCCTTCTCCTG). qPCR reactions were run in triplicate. The triplicate $\mathrm{Ct}$ values, were averaged, normalized against housekeeping genes HPRT and then compared against biological controls (untreated mice) using the $\Delta \Delta \mathrm{Ct}$ method of comparison. Fold expression was calculated assuming a doubling efficiency (2) per cycle. (Fold expression $=2^{-\Delta \Delta C t}$ ) 


\section{Flow Cytometry Analysis}

Single cell populations were isolated from vaginal tissue as previously described ${ }^{53}$. Briefly, vaginal tissue was minced into small pieces and digested first with Dispase II for 15 minutes and subsequently with a combination of DNase I $(.045 \mathrm{mg} / \mathrm{ml})$ and Collagenase $(2 \mathrm{mg} / \mathrm{ml})$ for 30 minutes. All digestions were carried out in a $37^{\circ} \mathrm{C}$ shaking water bath. All enzymes were obtained from Sigma-Aldrich. Cells were spun down and dead cells excluded using a live/dead stain (Molecular Probes, Thermo Fisher), then stained with appropriate antibodies, fixed with 1\% Paraformaldehyde (Electron Microscopy Sciences, PA) and run through an LSR II (BD, NJ) equipped with a UV laser. FlowJo software v9.8.2 (Tree Star, OR) was used to visualize and analyze cytometry data. Cells populations were analyzed as shown in the gating scheme (Supplementary Fig. 14).

\section{Antibodies}

The following antibodies were used for this study, all purchased from Biolegend unless specified otherwise: CD45(104), CD3e (145-2C11), CD11c (HL3, BD Biosciences), CD11b (M1/70), CD103 (2E7) XCR1 (ZET), SIRPa(P84), Gr1 (RB6-8C5, BD Biosciences), NK1.1(PK136), MHC class II I-A/I-E (M5/114.152), CD19 (6D5).

\section{Human Monocyte Isolation and Infection}

Peripheral blood mononuclear cells were obtained from the New York Blood Bank Center (NY) and monocytes isolated using a negative selection kit (Stemcell Technologies, MA). Monocytes were treated with PolyI:C (Sigma) or aminoglycosides at $2 \mu \mathrm{g} / \mathrm{ml}$ and $2 \mathrm{mg} / \mathrm{ml}$ respectively for 6 hours. Cells were washed once and infected with Influenza A virus strain $\mathrm{A} / \mathrm{PR} / 8 / 34$ (H1N1) at a multiplicity of infection of 2. Cells were incubated with the virus for 1 hour in $0.1 \%$ BSA in PBS. Cells were then washed once and incubated in DMEM containing $10 \%$ FBS, Pen/Strep, Sodium pyruvate and Hepes. RNA was isolated using an RNeasy extraction kit (Qiagen, CA) and viral RNA quantified using primers against Influenza A PR8 Polymerase A (FP:CGGTCCAAATTCCTGCTGAT; RP: CATTGGGTTCCTTCCATCCA).

\section{Viral Titer}

Vaginal washes from HSV-2 infected mice were collected in $50 \mu 1$ PBS and saved in $950 \mu 1$ PBS supplemented with $1 \%$ FBS, $10 \mathrm{mg} / \mathrm{ml}$ glucose $0.5 \mu \mathrm{M} \mathrm{MgCl}_{2}$ and $0.9 \mu \mathrm{M} \mathrm{CaCl}_{2}$. Washes were added in serial dilutions to a confluent monolayer of Vero cells and plaques visualized via crystal violet staining. ZIKV genome was quantified via qRT-PCR as previously described ${ }^{18}$. Briefly, cDNA prepared from vaginal washes was compared against a standard curve composed of purified ZIKV viral genomes. Primers against NS5 (F: GGCCACGAGTCTGTACCAAA; R: AGCTTCACTGCAGTCTTCC) were used to measure ZIKV RNA.

\section{In-vitro treatments}

Single cell splenocytes were plated at the density of $5 \times 10^{6}$ cells $/ \mathrm{ml}$ isolated and treated with kasugamycin $(2 \mathrm{mg} / \mathrm{ml}$ or $0.02 \mathrm{mg} / \mathrm{ml})$ and PolyI:C (High Molecular Weight, Invivogen,CA) $(2 \mu \mathrm{g} / \mathrm{ml}$ or $0.02 \mu \mathrm{g} / \mathrm{ml})$ for 6 hours. Mixtures of PolyI:C and kasugamycin were incubated 
together for 30 minutes at $37^{\circ} \mathrm{C}$ before addition to cells. In Supplementary Fig. 13, splenocytes were treated for 12 hours, washed 5 times, stained with cell trace violet (Thermo Fisher, CA), and incubated with splenic DCs isolated using PanDC separation kit (Stemcell Technologies, MA). After 6 hour incubation, cell trace violet dim and negative cells were sorted using an FACSAria (BD, NJ) into RLT buffer for RNA extraction. All in-vitro experiments were conducted with a minimum of three replicates and repeated twice.

\section{Statistics}

Gene expression data was analyzed using unpaired t-tests, assuming unequal standard deviation and correcting for multiple comparisons using the Holm-Sidak correction unless otherwise specified. Graphs depicting disease scores were analyzed using 2-way ANOVA with Holm-Sidak correction for multiple comparisons. Graphs depicting viral titers across a time course of infection were analyzed using 2-way ANOVA with no correction for multiple comparisons (Fisher's LSD) unless otherwise specified. Survival curves were analyzed using the log-rank (Mantel-Cox) test. Data from pooled experiments contained a control group within each experiment. $\mathrm{P}$ values not reported in the figures themselves can be found in Supplementary Table 2. All statistical analyses were two-sided and were performed in Graphpad Prism v7.0.

\section{Data Availability}

The data discussed in this publication have been deposited in NCBI's Gene Expression Omnibus $^{54}$ and are accessible through GEO Series accession number GSE94909 (https:// www.ncbi.nlm.nih.gov/geo/query/acc.cgi?acc=GSE94909).

\section{Supplementary Material}

Refer to Web version on PubMed Central for supplementary material.

\section{Acknowledgments}

We thank Dr. Yong Kong for his help analyzing the microarray data, and Huiping Dong for animal support. We thank Punya Biswal for help with visualizing the microarray data. This study was supported by funding from the NIH AI054359, R56AI125504, R01EB000487 and 1R21AI131284 (to AI). AI and AG are Investigator and Faculty Scholar of Howard Hughes Medical Institute. SG and MK are recipients of the James Hudson Brown - Alexander Brown Coxe Postdoctoral Fellowships at Yale University.

\section{References}

1. Badal S, Her YF, Maher LJ. Nonantibiotic Effects of Fluoroquinolones in Mammalian Cells. J Biol Chem. 2015; 290:22287-22297. [PubMed: 26205818]

2. Kalghatgi S, et al. Bactericidal antibiotics induce mitochondrial dysfunction and oxidative damage in Mammalian cells. Science Translational Medicine. 2013; 5:192ra85.

3. Moullan N, et al. Tetracyclines Disturb Mitochondrial Function across Eukaryotic Models: A Call for Caution in Biomedical Research. CellReports. 2015; 10:1681-1691.

4. Yang JH, et al. Antibiotic-Induced Changes to the Host Metabolic Environment Inhibit Drug Efficacy and Alter Immune Function. Cell Host \& Microbe. 2017; :1-13. DOI: 10.1016/j.chom. 2017.10.020 
5. Linehan MM, et al. In Vivo Role of Nectin-1 in Entry of Herpes Simplex Virus Type 1 (HSV-1) and HSV-2 through the Vaginal Mucosa. Journal of Virology. 2004; 78:2530-2536. [PubMed: 14963155]

6. Shin H, Iwasaki A. Generating protective immunityagainst genital herpes. Trends in Immunology. 2013; 34:487-494. [PubMed: 24012144]

7. Corey L, Schiffer JT. Rapid host immune response and viral dynamics in herpes simplex virus-2 infection. Nature Medicine. 2013; 19:280-290.

8. Khoury-Hanold W, et al. Viral Spread to Enteric Neurons Links Genital HSV-1 Infection to Toxic Megacolon and Lethality. Cell Host \& Microbe. 2016; 19:788-799. [PubMed: 27281569]

9. McDermott MR, et al. Immunity in the female genital tract after intravaginal vaccination of mice with an attenuated strain of herpes simplex virus type 2. Journal of Virology. 1984; 51:747-753. [PubMed: 6088797]

10. Busscher GF, Rutjes FPJT, van Delft FL. 2-Deoxystreptamine: Central Scaffold of Aminoglycoside Antibiotics $\uparrow$. Chem Rev. 2005; 105:775-792. [PubMed: 15755076]

11. Wilson DN. Ribosome-targeting antibiotics and mechanisms of bacterial resistance. Nat Rev Micro. 2014; 12:35-48.

12. Morgun A, et al. Uncovering effects of antibiotics on the host and microbiota using transkingdom gene networks. Gut. 2015; 64:1732-1743. [PubMed: 25614621]

13. Meier E, et al. A family of interferon-induced Mx-related mRNAs encodes cytoplasmic and nuclear proteins in rat cells. Journal of Virology. 1988; 62:2386-2393. [PubMed: 3373571]

14. Staeheli P, Grob R, Meier E, Sutcliffe JG, Haller O. Influenza virus-susceptible mice carry Mx genes with a large deletion or a nonsense mutation. Mol Cell Biol. 1988; 8:4518-4523. [PubMed: 2903437]

15. Haller O, Staeheli P, Schwemmle M, Kochs G. Mx GTPases: dynamin-like antiviral machines of innate immunity. Trends Microbiol. 2015; 23:154-163. [PubMed: 25572883]

16. Ichinohe $\mathrm{T}$, et al. Microbiota regulates immune defense against respiratory tract influenza A virus infection. Proc Natl Acad Sci USA. 2011; 108:5354-5359. [PubMed: 21402903]

17. Grimm D, et al. Replication fitness determines high virulence of influenza A virus in mice carrying functional Mx1 resistance gene. Proc Natl Acad Sci U S A. 2007; 104:6806-6811. [PubMed: 17426143]

18. Yockey LJ, et al. Vaginal Exposure to Zika Virus during Pregnancy Leads to Fetal Brain Infection. Cell. 2016; 166:1247-1256.e4. [PubMed: 27565347]

19. Yamamoto M, et al. Role of adaptor TRIF in the MyD88-independent toll-like receptor signaling pathway. Science. 2003; 301:640-643. [PubMed: 12855817]

20. Honda K, et al. IRF-7 is the master regulator of type-I interferon-dependent immune responses. Nature. 2005; 434:772-777. [PubMed: 15800576]

21. Schneider WM, Chevillotte MD, Rice CM. Interferon-Stimulated Genes: A Complex Web of Host Defenses. Annu Rev Immunol. 2014; 32:513-545. [PubMed: 24555472]

22. Schmid S, Mordstein M, Kochs G, Garcia-Sastre A, tenOever BR. Transcription Factor Redundancy Ensures Induction of the Antiviral State. J Biol Chem. 2010; 285:42013-42022. [PubMed: 20943654]

23. Iijima N, Iwasaki A. T cell memory. A local macrophage chemokine network sustains protective tissue-resident memory CD4 T cells. Science. 2014; 346:93-98. [PubMed: 25170048]

24. Shortman K, Heath WR. The CD8+ dendritic cell subset. Immunol Rev. 2010; 234:18-31. [PubMed: 20193009]

25. Schulz O, et al. Toll-like receptor 3 promotes cross-priming to virus-infected cells. Nature. 2005; 433:887-892. [PubMed: 15711573]

26. Heng TSP, Painter MW. Immunological Genome Project Consortium. The Immunological Genome Project: networks of gene expression in immune cells. Nat Immunol. 2008; 9:1091-1094. [PubMed: 18800157]

27. Miller JC, et al. Deciphering the transcriptional network of the dendritic cell lineage. Nat Immunol. 2012; :1-14. DOI: $10.1038 /$ ni. 2370 
28. Crozat K, et al. Cutting Edge: Expression of XCR1 Defines Mouse Lymphoid-Tissue Resident and Migratory Dendritic Cells of the CD8 + Type. journal of immunology. 2011; 187:4411-4415.

29. Yamazaki C, et al. Critical roles of a dendritic cell subset expressing a chemokine receptor, XCR1. The Journal of Immunology. 2013; 190:6071-6082. [PubMed: 23670193]

30. Begg EJ, Barclay ML. Aminoglycosides--50 years on. Br J Clin Pharmacol. 1995; 39:597-603. [PubMed: 7654476]

31. Rizzi MD, Hirose K. Aminoglycoside ototoxicity. Curr Opin Otolaryngol Head Neck Surg. 2007; 15:352-357. [PubMed: 17823553]

32. Matsui JI, Gale JE, Warchol ME. Critical signaling events during the aminoglycoside-induced death of sensory hair cellsin vitro. J Neurobiol. 2004; 61:250-266. [PubMed: 15389694]

33. Cheng AG, Cunningham LL, Rubel EW. Mechanisms of hair cell death and protection. Curr Opin Otolaryngol Head Neck Surg. 2005; 13:343-348. [PubMed: 16282762]

34. Wargo KA, Edwards JD. Aminoglycoside-Induced Nephrotoxicity. Journal of Pharmacy Practice. 2014; 27:573-577. [PubMed: 25199523]

35. Laurent G, Kishore BK, Tulkens PM. Aminoglycoside-induced renal phospholipidosis and nephrotoxicity. Biochem Pharmacol. 1990; 40:2383-2392. [PubMed: 2268362]

36. Ryu DH, Rando RR. Aminoglycoside binding to human and bacterial A-Site rRNA decoding region constructs. Bioorg Med Chem. 2001; 9:2601-2608. [PubMed: 11557348]

37. Recht MI, Fourmy D, Blanchard SC, Dahlquist KD, Puglisi JD. RNA sequence determinants for aminoglycoside binding to an A-site rRNA model oligonucleotide. J Mol Biol. 1996; 262:421436. [PubMed: 8893854]

38. Walter F, Vicens Q, Westhof E. Aminoglycoside-RNA interactions. Current Opinion in Chemical Biology. 1999; 3:694-704. [PubMed: 10600721]

39. Rausch K, et al. Screening Bioactives Reveals Nanchangmycin as a Broad Spectrum Antiviral Active against Zika Virus. CellReports. 2017; 18:804-815.

40. Retallack H, et al. Zika virus cell tropism in the developing human brain and inhibition by azithromycin. Proc Natl Acad Sci USA. 2016; 113:14408-14413. [PubMed: 27911847]

41. Porter JD, et al. Identification of novel macrolides with antibacterial, anti-inflammatory and type I and III IFN-augmenting activity in airway epithelium. J Antimicrob Chemother. 2016; 71:27672781. [PubMed: 27494903]

42. Sistigu A, et al. Cancer cell-autonomous contribution of type I interferon signaling to the efficacy of chemotherapy. Nature Medicine. 2014; 20:1301-1309.

43. Hashino E, Shero M. Endocytosis of aminoglycoside antibiotics in sensory hair cells. Brain Research. 1995; 704:135-140. [PubMed: 8750975]

44. Myrdal SE, Steyger PS. TRPV1 regulators mediate gentamicin penetration of cultured kidney cells. Hearing Research. 2005; 204:170-182. [PubMed: 15925202]

45. Assas BM, Wakid MH, Zakai HA, Miyan JA, Pennock JL. Transient receptor potential vanilloid 1 expression and function in splenic dendritic cells: a potential role in immune homeostasis. Immunology. 2016; 147:292-304. [PubMed: 26643862]

46. Moestrup SK, et al. Evidence that epithelial glycoprotein 330/megalin mediates uptake of polybasic drugs. J Clin Invest. 1995; 96:1404-1413. [PubMed: 7544804]

47. Christensen EI, Birn H. Megalin and cubilin: multifunctional endocytic receptors. Nat Rev Mol Cell Biol. 2002; 3:258-268.

48. Iyoda T, et al. The CD8 +Dendritic Cell Subset Selectively Endocytoses Dying Cells in Culture and In Vivo. J Exp Med. 2002; 195:1289-1302. [PubMed: 12021309]

49. Bedoui S, et al. Cross-presentation of viral and self antigens by skin-derived CD103+ dendritic cells. Nat Immunol. 2009; 10:488-495. [PubMed: 19349986]

50. Hong S, et al. Evidence That Antibiotics Bind to Human Mitochondrial Ribosomal RNA Has Implications for Aminoglycoside Toxicity. J Biol Chem. 2015; 290:19273-19286. [PubMed: 26060252]

51. Sun Q, et al. The specific and essential role of MAVS in antiviral innate immune responses. Immunity. 2006; 24:633-642. [PubMed: 16713980] 
52. Horisberger MA, Staeheli P, Haller O. Interferon induces a unique protein in mouse cells bearing a gene for resistance to influenza virus. Proc Natl Acad Sci U S A. 1983; 80:1910-1914. [PubMed: 6188159]

53. Shin $\mathrm{H}$, Iwasaki A. A vaccine strategy that protects against genital herpes by establishing local memory T cells. Nature. 2012; 491:463-467. [PubMed: 23075848]

54. Edgar R, Domrachev M, Lash AE. Gene Expression Omnibus: NCBI gene expression and hybridization array data repository. Nucleic Acids Research. 2002; 30:207-210. [PubMed: 11752295] 

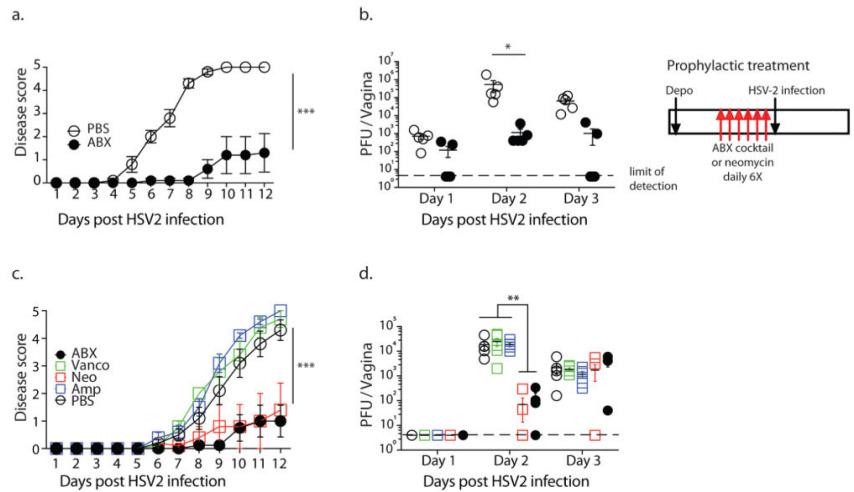

$$
\text { d. }
$$
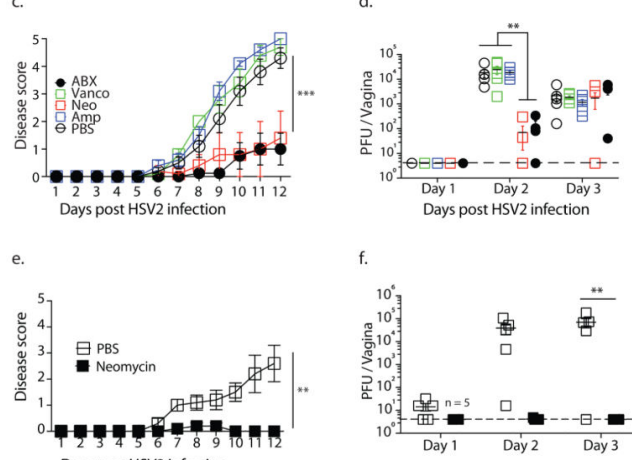

f.
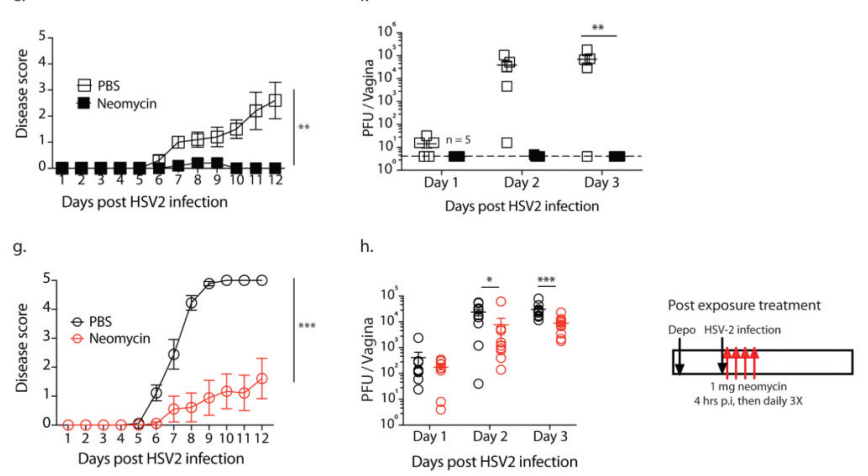

Figure 1. Vaginal application of neomycin confers prophylactic and post exposure antiviral protection against HSV-2 in microbiota-independent manner

Conventional $(\mathrm{a}-\mathrm{d}, \mathrm{g}-\mathrm{h})$ and germ-free $(\mathrm{e}, \mathrm{f})$ mice were treated subcutaneously with DepoProvera and five days later were inoculated intravaginally with an antibiotic cocktail $(a, b)$ or singly with the indicated antibiotic ( $\mathrm{c}-\mathrm{f})$ daily for 6 days $(\mathrm{n}=5$ mice per group). After 6 days of treatment, all mice were infected intravaginally with HSV-2. For post exposure neomycin treatment, depo-treated mice $(n=9-10$, data pooled from two independent experiments) were infected with HSV-2, then treated with $1 \mathrm{mg}$ neomycin or PBS at $4 \mathrm{hrs,}$ $24 \mathrm{hrs}, 48 \mathrm{hrs}$ and $72 \mathrm{hrs}$ after infection $(\mathrm{g}, \mathrm{h})$. For all infected mice, disease score was monitored daily (a,c,e,g) and vaginal wash collected on the first three days (b,d,f,h). All error bars represent SEM. Significance was calculated using 2-way ANOVA with HolmSidak correction for multiple comparisons (a,c,e,g) or with Fisher's LSD test (b,d,f,h). * represents $\mathrm{p}$ values $<0.05$ and exact $\mathrm{p}$ values are reported in Supplementary Table 2. Experiments in a,b,g\&h were repeated independently with similar results. 


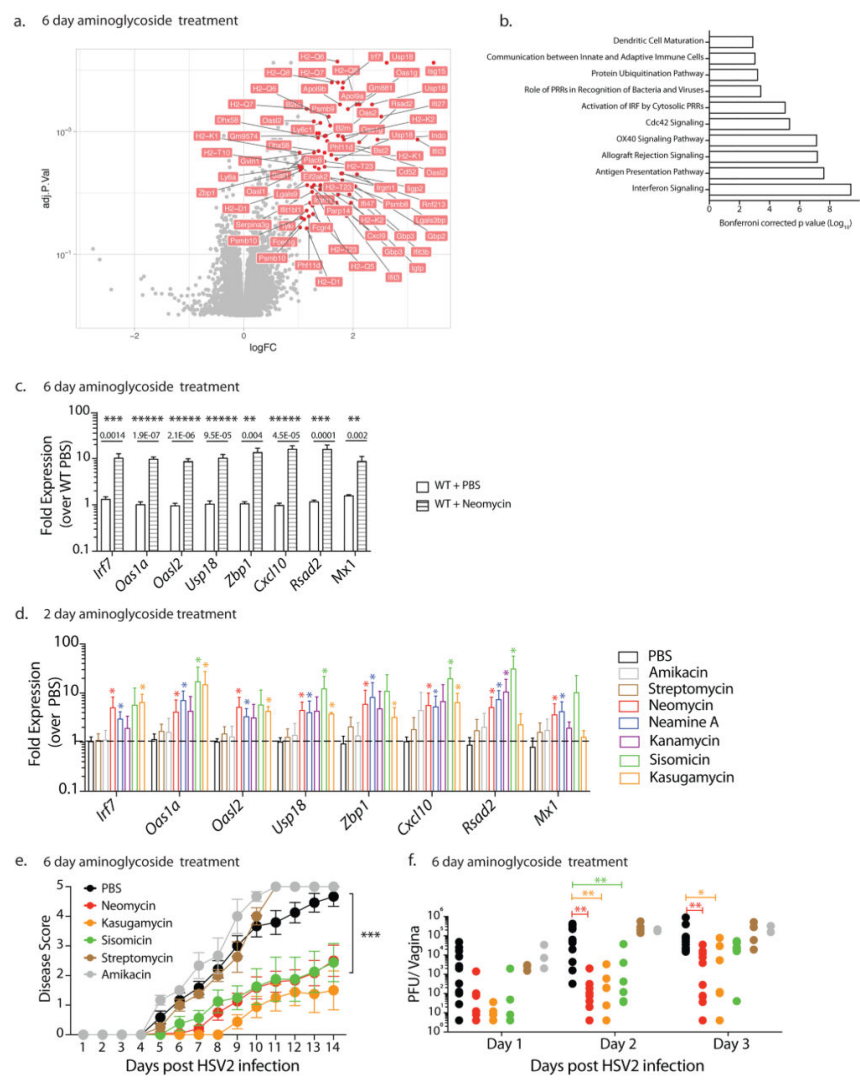

Figure 2. Vaginal application of most aminoglycosides induce interferon-stimulated genes, which is linked to antiviral protection

Microarray analysis of vaginal gene expression in neomycin-treated mice were normalized against PBS-treated mice ( $\mathrm{n}=3$ per group) and fold expression and $\mathrm{p}$ values plotted (a).

Significantly differentially expressed known genes (fold expression $>2 X$, p value $<0.05$ ) are labeled. Ingenuity pathway analysis was used to identify the top ten signaling pathways enriched upon neomycin treatment (b). In an independent experiment Depo-treated mice were treated with neomycin or PBS ( $n=9$, data pooled from two independent experiments) and vaginal gene expression measured via qPCR with error bars representing SEM (c). Depo-treated mice were inoculated intravaginally with the indicated aminoglycosides ( $1 \mathrm{mg} /$ day) for 2 days ( $n=3-6$ mice per group, data pooled from two independent experiments). Vaginal gene expression was analyzed via qPCR and all comparisons were made against PBS samples with error bars representing SD (d). Statistical significance of gene expression data was calculated using unpaired t-tests with Holm-Sidak correction for multiple comparisons with * representing p values $<0.05(\mathrm{c}, \mathrm{d})$. Depo-treated mice $(\mathrm{n}=3-12$, data pooled from 3 independent experiments) were treated intravaginally with $1 \mathrm{mg}$ of the indicated aminoglycosides daily for 6 days and then infected with HSV-2 (e,f). Disease score was monitored (e) and vaginal viral titers measured (f). Error bars represent SEM and statistical significance was calculated using 2-way ANOVA with Holm-Sidak correction for multiple comparisons. Exact $\mathrm{p}$ values for all comparisons are reported in Supplementary Table 2 . 
a.

1 day aminoglycoside treatment - lung ISG expression

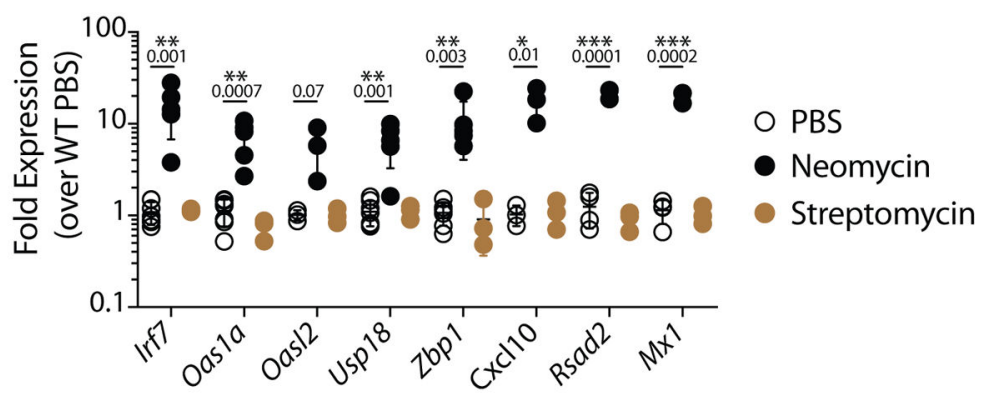

c. ZIKV

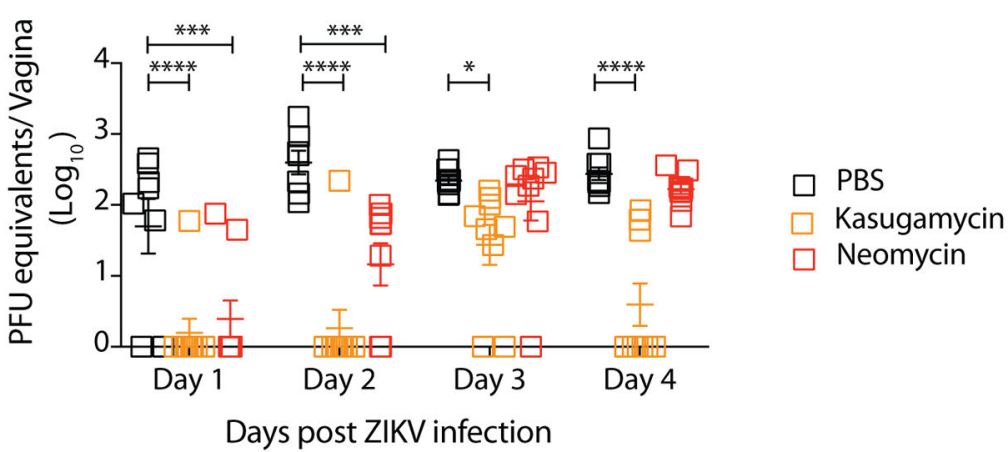

b.

Highly virulent influenza A virus

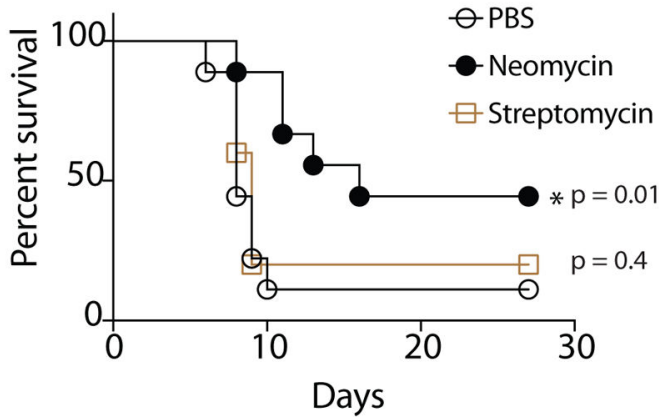

d.

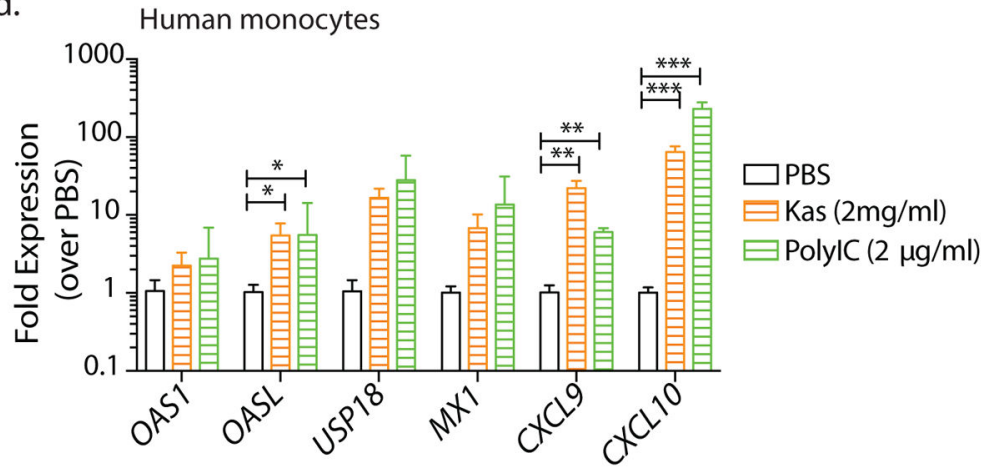

e.

Influenza A virus

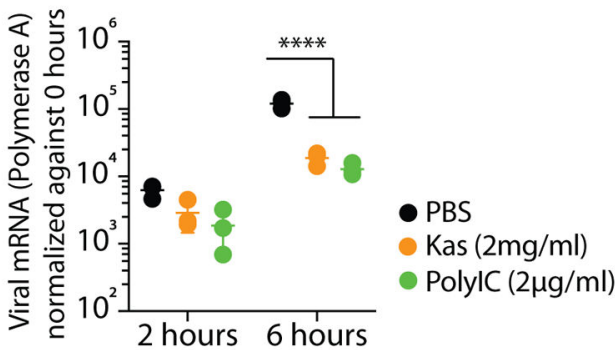

Figure 3. Aminoglycosides confer broad protection against both RNA and DNA viruses Mice were treated intranasally with $2 \mathrm{mgs}$ neomycin or streptomycin (data pooled from two independent experiments, one with PBS and Neomycin groups $(n=4)$ and the second which contained PBS, Neomycin and Streptomycin treatment groups $(n=3))$ and gene expression analyzed 24 hours later in lung tissue (a). Error bars represent SD and significance was calculated using multiple t-tests with Holm-Sidak correction for multiple comparisons. Mx 1 congenic mice (data pooled from 2 independent experiments, one which contained PBS and Neomycin groups $(n=4)$ and the second with PBS, Neomycin and Streptomycin treatment groups $(n=5))$ were pretreated intranasally with the indicated aminoglycoside (2mgs) or PBS and infected 24 hours later with highly virulent influenza strain PR8 and survival curves compared using a log rank (Mantel-Cox) test (b). Depo-treated mice $(n=7-8$, data pooled 
from two independent experiments) received intravaginal aminoglycoside or PBS daily for 6 days and were infected with 25,000 PFU ZIKV intravaginally and vaginal viral titers were calculated via qPCR. Error bars represent SEM and significance was calculated using 2-way ANOVA with Fisher's LSD test. (c). Primary human monocytes were treated with kasugamycin $(2 \mathrm{mg} / \mathrm{ml})$ or Poly I:C $(2 \mu \mathrm{g} / \mathrm{ml})$. Six hours after treatment, gene expression was analyzed ( $n=3$ replicates per treatment). Error bars represent SD and significance was calculated using multiple t-tests with Holm-Sidak correction for multiple comparisons (d). In a separate experiment, kasugamycin-treated monocytes were infected with Influenza A/PR/8/34 (H1N1) strain at a multiplicity of infection of 2 and RNA collected 2 and 6 hours post infection ( $n=3$ replicates per treatment). Virus levels were quantified via qPCR using primers against Polymerase A. Error bars represent SD and significance was calculated using 2-way ANOVA with Fisher's LSD test (e). * represents p values $<0.05$ and exact $p$ values are reported in Supplementary Table 2. Experiments shown in d,e were repeated independently with similar results. 
a. 6 day treatment

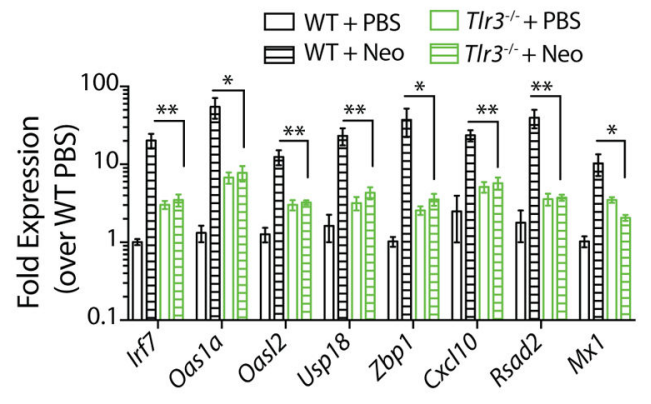

d. 6 day treatment

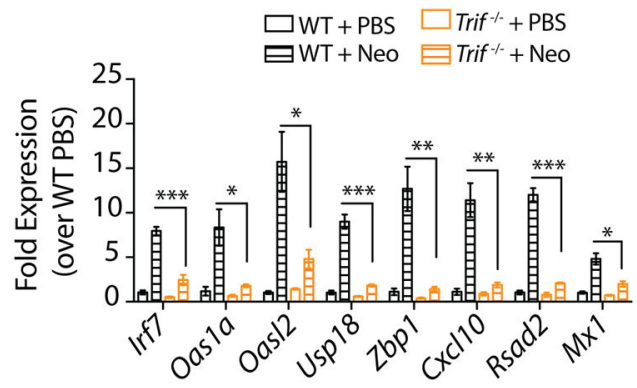

g. 6 day treatment

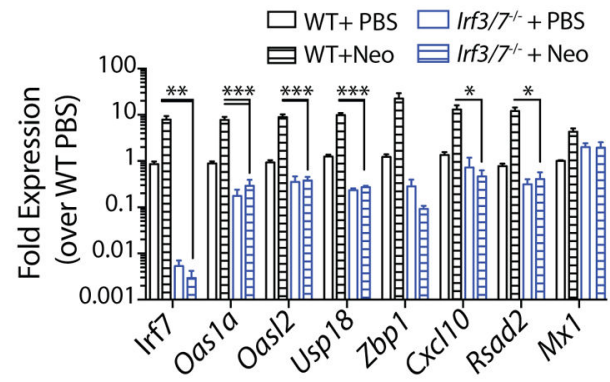

b.

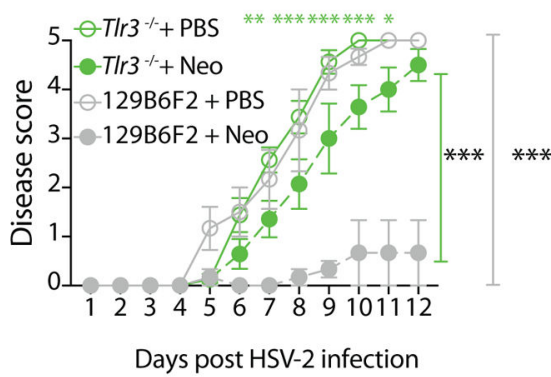

e.

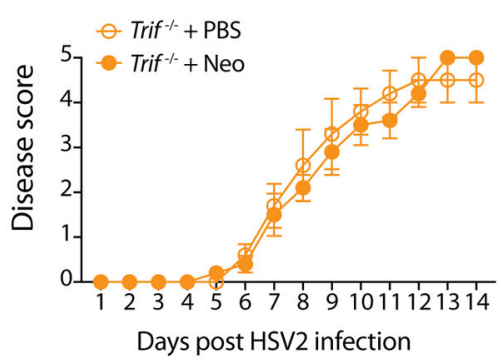

h.

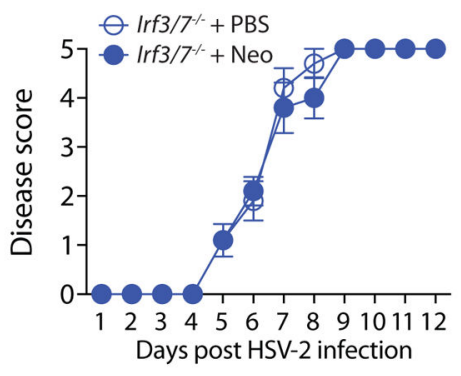

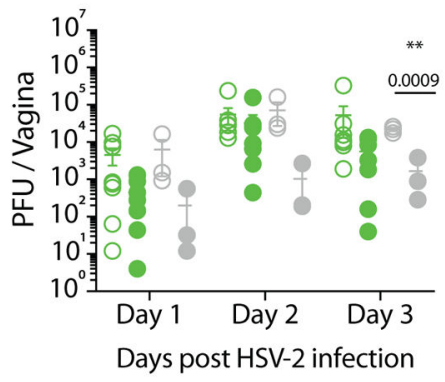

f.

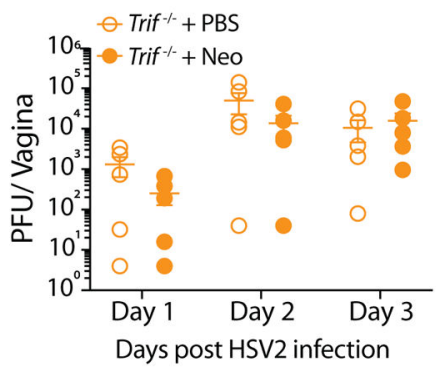

i.

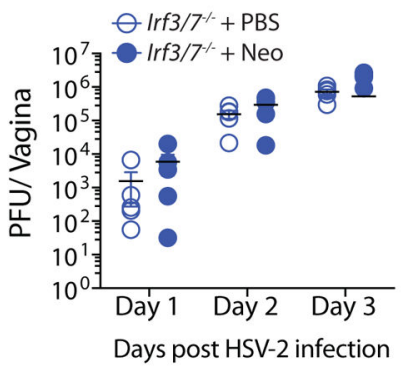

Figure 4. Aminoglycosides mediate antiviral immunity via the TLR3-TRIF-IRF3/7 signaling pathway

Five days after Depo treatment wildtype and knockout mice of the indicated genotypes ( $\mathrm{n}=3-5$ mice per group) were treated with $1 \mathrm{mg}$ neomycin intravaginally, daily for 6 days and vaginal gene expression measured $(\mathrm{a}, \mathrm{d}, \mathrm{g})$. In an independent experiment these mice $(\mathrm{n}=3-5)$ were infected with HSV-2, disease scores $(b, e, h)$ and vaginal viral titers measured $(c, f, i)$. $129 \mathrm{~S} 1 \times \mathrm{B} 6 \mathrm{~F} 2$ mice were used as wild type controls for TLR ${ }^{-1-}$ mice $(\mathrm{b}, \mathrm{c})$ and $\mathrm{C} 57 \mathrm{BL} / 6 \mathrm{~N}$ mice were used as wildtype controls for remaining (a,d-i). Error bars represent SEM. Significance was calculated using 2-way ANOVA with Holm-Sidak test for multiple comparisons $(b, e, h)$ or unpaired t-tests $(\mathrm{c}, \mathrm{f}, \mathrm{i})$ and unpaired t-tests correcting for multiple comparisons $(\mathrm{a}, \mathrm{d}, \mathrm{g})$. * represents $\mathrm{p}$ values $<0.05$ and exact $\mathrm{p}$ values are reported in Supplementary Table 2. Experiments shown in a-d, g-i were repeated independently with similar results. 


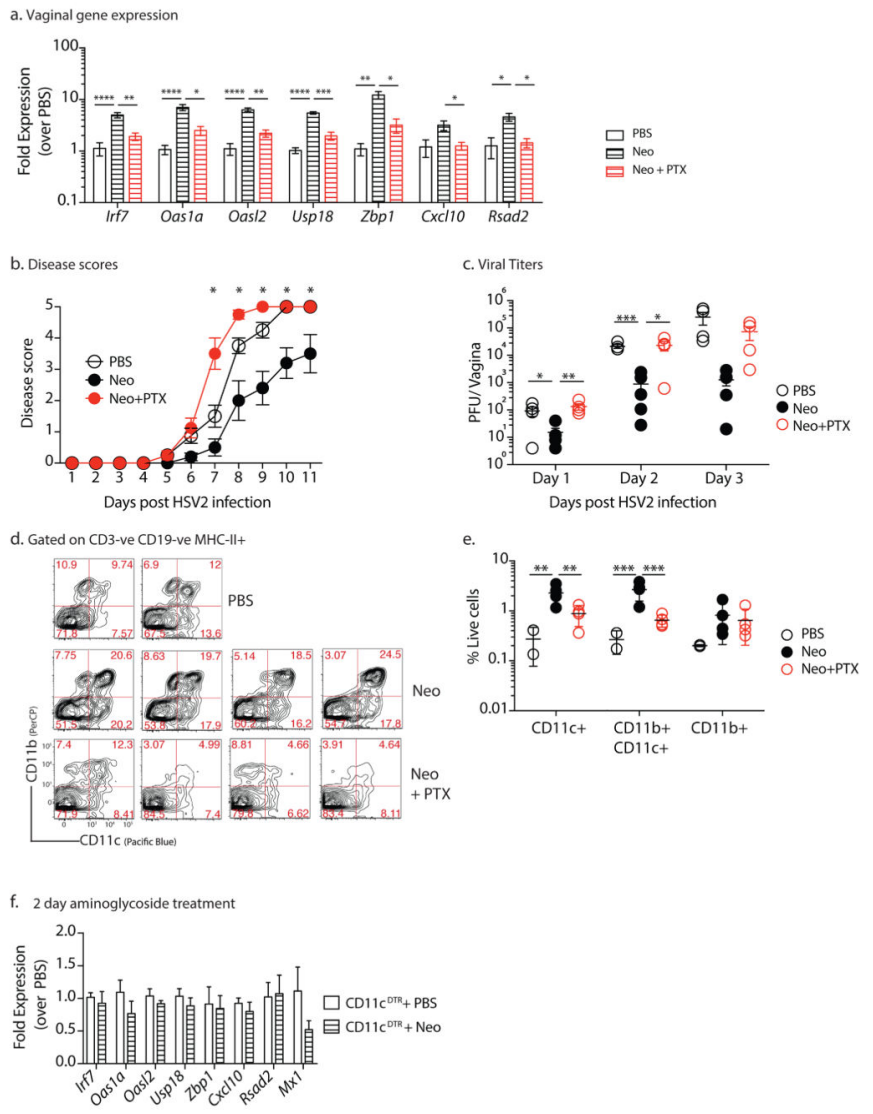

Figure 5. Recruited dendritic cells are required for ISG induction by neomycin Mice treated subcutaneously with Depo-Provera were inoculated intravaginally with neomycin (1mg), PBS daily for 6 days ( $\mathrm{n}=4$ mice per group) or neomycin $(1 \mathrm{mg})$ and pertussis toxin (PTX) $(0.5 \mu \mathrm{g})$ daily. After 1 week of treatment, vaginal gene expression was quantified, error bars represebt SEM and significance was calculated using t-tests and correcting for multiple comparisons (a). In an independent experiment, neomycin and PTX treated mice ( $\mathrm{n}=4-5)$ were infected with HSV-2 and disease score monitored daily (b) and vaginal viral titers measured (c). Error bars represent SEM and significance was calculated using 2-way ANOVA correcting for multiple comparisons (b) or with Fisher's LSD test (c). Vaginal dendritic cell populations were analyzed via flow cytometry $(n=2-4)$, plots are gated on CD19-,CD3-, Gr1-, MHCII+ (d) and quantified in (e) (gating schema in Supplementary Fig. 14). Error bars represent SD and significance was calculated using 2way ANOVA with Fisher's LSD test. Experiments shown in d,e were repeated independently with similar results. Depo-Provera treated CD11cDTR mice were treated with $125 \mathrm{ng}$ diphtheria toxin to deplete dendritic cells, treated intravaginally with $1 \mathrm{mg}$ neomycin for two days ( $\mathrm{n}=8$, data pooled from two independent experiments) and vaginal gene expression measured via qPCR, error bars represent SEM (f). Significance was calculated using unpaired t-tests, correcting for multiple comparisons. * represent $\mathrm{p}$ values $<0.05$ and exact $\mathrm{p}$ values for all comparisons are reported in Supplementary Table 2 
a. Gated on CD3-CD19-Gr1-NK1.1-MHC II+CD11b-ve CD11C+

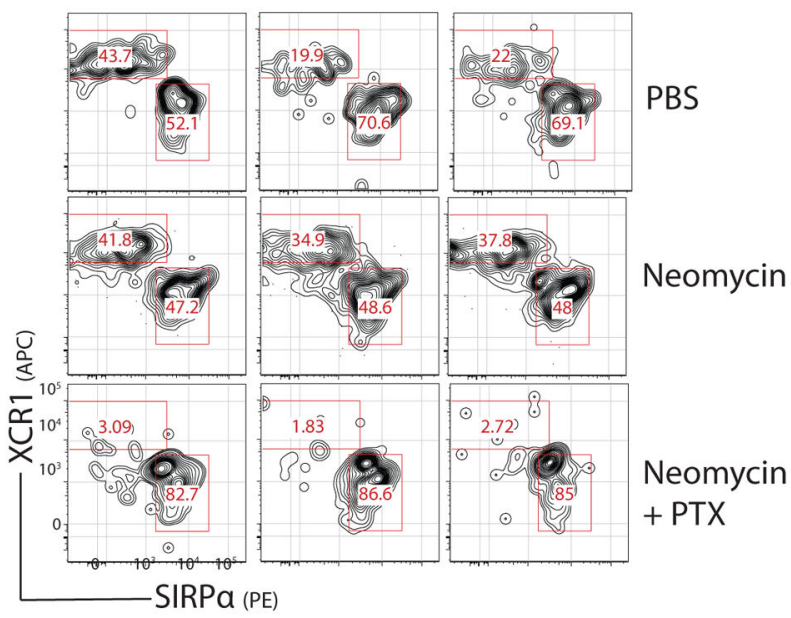

b.

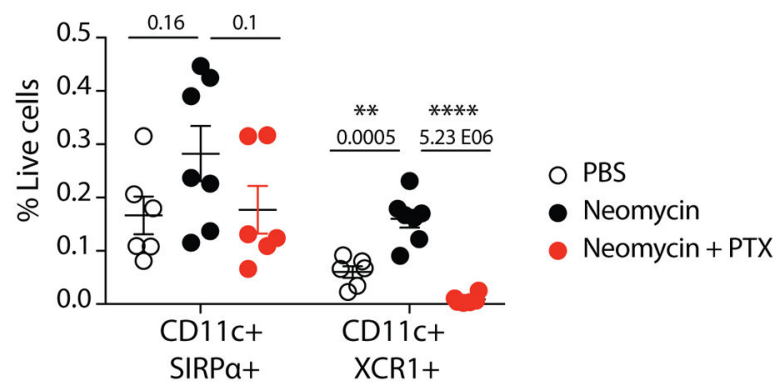

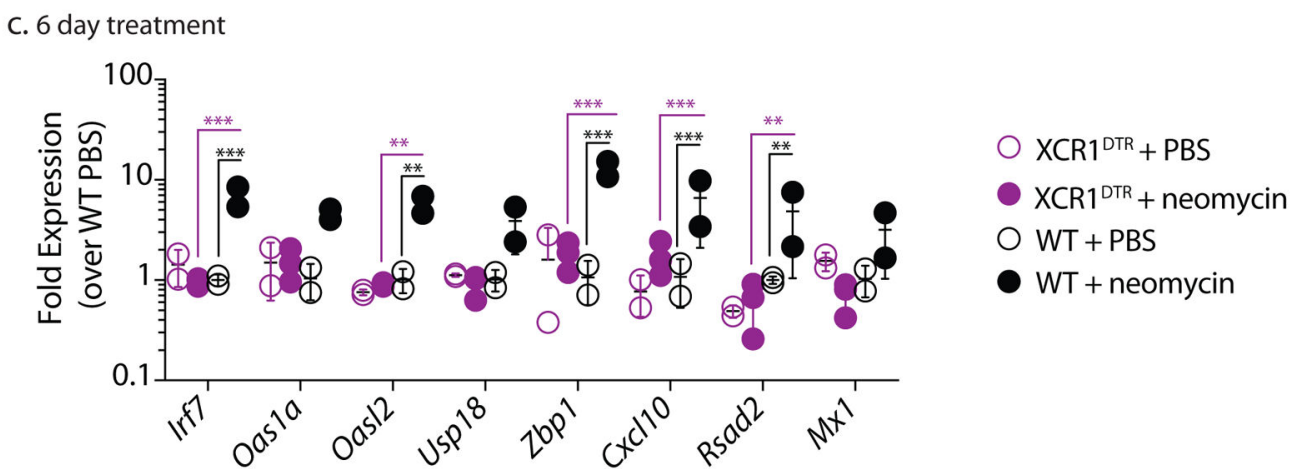

Figure 6. Recruited XCR1+ dendritic cells are required for ISG induction

Mice treated subcutaneously with Depo-Provera were inoculated intravaginally with neomycin (1mg), PBS daily for 1 week or neomycin (1mg) and pertussis toxin (PTX) $(0.5 \mu \mathrm{g})$ daily. After 1 week of treatment, recruitment of XCR1+ vaginal DCs to the vaginal mucosa was measured. Representative FACS plots from one experiment $(n=3)$ are gated on CD19-, CD3-, Gr1-, MHCII+CD11c+ CD11b- live cells (a). Frequency of DCs was quantified as a frequency of live cells $(n=6-7)(b)$. Data were pooled from two independent experiments, error bars represent SEM and significance was calculated using unpaired ttests. Depo-treated XCR1 ${ }^{\text {DTR }}$ mice were treated with 500ng diphtheria toxin to deplete XCR1+dendritic cells, treated intravaginally with $1 \mathrm{mg}$ neomycin for two days $(\mathrm{n}=2-5)$. Vaginal gene expression measured via qPCR, error bars represent SD and significance was calculated using 2-way ANOVA with Holm-Sidak correction for multiple comparisons (c). * represent $\mathrm{p}$ values $<0.05$ and exact $\mathrm{p}$ values for all comparisons are reported in Supplementary Table 2 